\title{
Single Frame Fringe Pattern Analysis for Phase Recovery with Analytic Signal
}

\author{
Khalid Assid, Vamara Dembele, Faiçal Alaoui, and Abdel Karim Nassim \\ Laboratoire Instrumentation de Mesure et de Contrôle, Equipe Metrologie Optique et Traitement Image, \\ Université Chouaib Doukkali, Faculté des Sciences, BP 20, El Jadida 24000, Morocco \\ Correspondence should be addressed to Khalid Assid, khalidassid@gmail.com
}

Received 25 March 2012; Accepted 9 May 2012

Academic Editors: O. Frazao and D. Poitras

Copyright (c) 2012 Khalid Assid et al. This is an open access article distributed under the Creative Commons Attribution License, which permits unrestricted use, distribution, and reproduction in any medium, provided the original work is properly cited.

We consider a new application of the normalized Hilbert-Huang transform to extract directly the phase from a single fringe pattern. We present a technique to provide, with good accuracy, the phase distribution from a single interferogram without unwrapping step and this by a new exploitation of the analytic signal corresponding to each intrinsic mode function, resulting from onedimensional empirical mode decomposition of the fringe pattern. A theoretical analysis was carried out for this technique, followed by computer simulations and a real experimental fringe pattern for verification.

\section{Introduction}

In optical techniques, several physical magnitudes in a variety of scientific and engineering fields are encoded as the phase of a periodic intensity profile. The developments of more sophisticated phase evaluation algorithms are continuously needed $[1,2]$.

The use of a single fringe pattern for phase retrieval is more suitable in dynamic processes. The unwrapping step, which provides, a continuous phase distribution over its definition domain implies several difficulties and sophisticated algorithms are then needed [3].

Recently, the normalized Hilbert-Huang transform (NHHT) has emerged as a promising and powerful tool for processing nonlinear and nonstationary signals. It consists of three parts: empirical mode decomposition (EMD), intrinsic mode functions (IMF) normalization, and Hilbert spectral analysis (HSA). The NHHT was developed by Huang et al. [4-7] to overcome the limitations set by the Bedrosian and Nuttall theorems when applying the regular Hilbert transform [8-12].

In our work, we attempt to retrieve the unwrapped phase from a single image. Our fringe analysis technique consists of decomposing the fringe pattern into IMFs, applying the HSA to each normalized IMF, then constructing individual analytic signals and computing the phase gradient. This leads directly to the phase distribution avoiding the complex step of phase unwrapping.

Computer simulation results obtained using our method are in very good agreement with those produced using phaseshifting methods [13]. To validate the performance of the proposed technique, it has been further tested on experimental fringe patterns. It was shown that the result totally agrees with that retrieved by phase-shifting techniques.

\section{Empirical Mode Decomposition for Fringe Pattern Analysis}

The fringe pattern, derived from a two-beam interferometer is characterized by the sinusoidal dependence of the intensity on the spatial coordinates $(x, y)$ of the image plane $[1,2]$ :

$$
I(x, y)=a(x, y)+b(x, y) \cos \phi(x, y),
$$

where $a(x, y)$ is the background. The terms $b(x, y)$ and $\cos \phi(x, y)$ are the modulation intensity and the optical phase.

The empirical mode decomposition (EMD) analysis is an adaptive and fully driven data method to decompose any signal into a set of components called intrinsic mode functions (IMF) and a residue [5]. Adding all the IMFs 
together with the residue reconstructs the original signal without information loss or distortion [14]:

$$
I(x)=\sum_{k=1}^{n-1} \operatorname{IMF}_{k}(x)+\operatorname{Res}(x) .
$$

Adding all the IMFs by just skipping the DC component that is, $\operatorname{Res}(x)$, we remove the background. The filtered fringe pattern is

$$
J(x)=b(x) \cos \phi(x)=\sum_{k=1}^{n-1} \operatorname{IMF}_{k}(x) .
$$

When decomposing the intensity by EMD, the resulting IMFs are not restricted to narrow band signals and can be both amplitude and frequency modulated [8]. Each IMF should be representable in the form

$$
\operatorname{IMF}_{k}(x)=b_{k}(x) \cos \left[\phi_{k}(x)\right]
$$

where the amplitude $b_{k}(x)$ and phase $\phi_{k}$ are both physically and mathematically meaningful. Once a suitable polar parameterization is determined, it is possible to analyse $J(x)$ by processing these individual components. The most common procedure to determine a polar representation is the analytic method of signal processing [15].

\section{Analytic Signal Construction}

To construct the analytic signal corresponding to each IMF, the simplest way is by employing the Hilbert transform [6]. The real part of an analytic signal is the original input, whereas its imaginary component is the Hilbert transform HT of that signal.

For each $\operatorname{IMF}_{k}(x)$, the corresponding analytic signal is

$$
Z_{k}(x)=\operatorname{IMF}_{k}(x)+i \operatorname{HT}\left[\operatorname{IMF}_{k}(x)\right] .
$$

Using the exponential notation

$$
Z_{k}(x)=b_{k}(x) e^{i \phi_{k}(x)}
$$

the following instantaneous attributes of $Z_{k}(x)$ can be defined:

$$
\begin{gathered}
b_{k}(x)=\left\{\operatorname{IMF}_{k}^{2}(x)+\operatorname{HT}\left[\operatorname{IMF}_{k}(x)\right]^{2}\right\}^{1 / 2}, \\
\operatorname{IMFN}_{k}(x)=\frac{\operatorname{IMF}_{k}(x)}{b_{k}(x)} \\
\phi_{k}(x)=\operatorname{artctan}\left\{\frac{b_{k} \cdot \operatorname{HT}\left[\operatorname{IMFN}_{k}(x)\right]}{\operatorname{IMF}_{k}(x)}\right\} \\
f_{k}(x)=\frac{1}{2 \pi} \frac{d \phi_{k}(x)}{d x},
\end{gathered}
$$

where $b_{k}(x)$ is the instantaneous amplitude, $\operatorname{IMFN}_{k}(x)$ is the normalized $\operatorname{IMF}_{k}(x), \phi_{k}(x)$ is the instantaneous phase, and $f_{k}(x)$ is the instantaneous frequency [16].

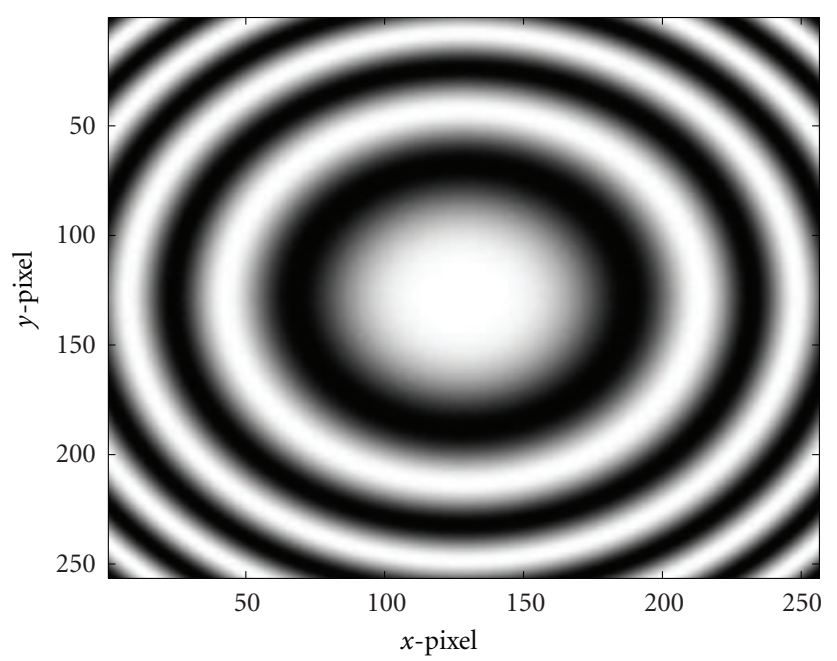

Figure 1: The fringe pattern $I$.

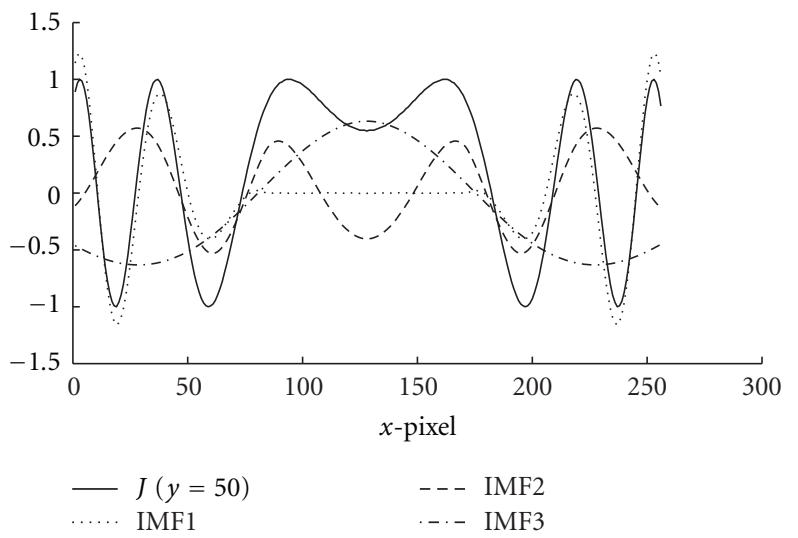

Figure 2: The tree IMFs component resulting from EMD decomposition of the data row $J(y=50)$.

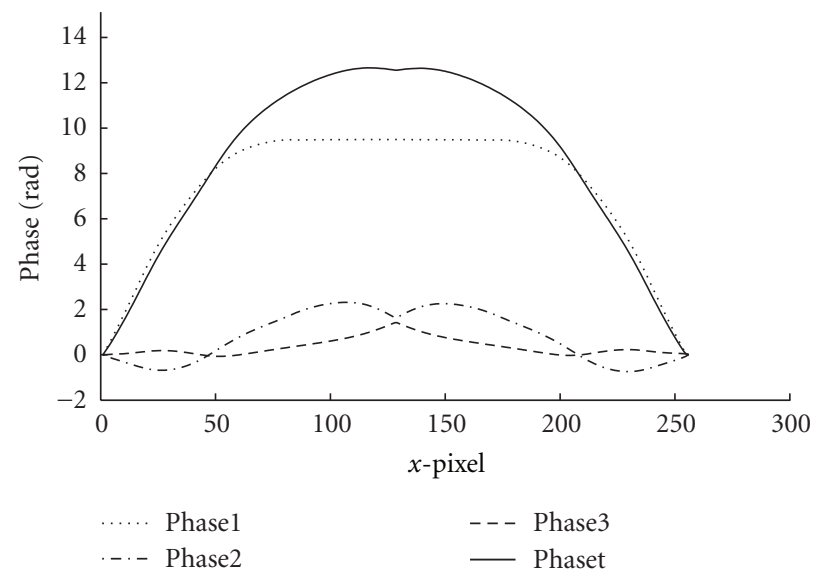

FIGURE 3: The individual contribution of each IMF to construct the global phase phaset. 


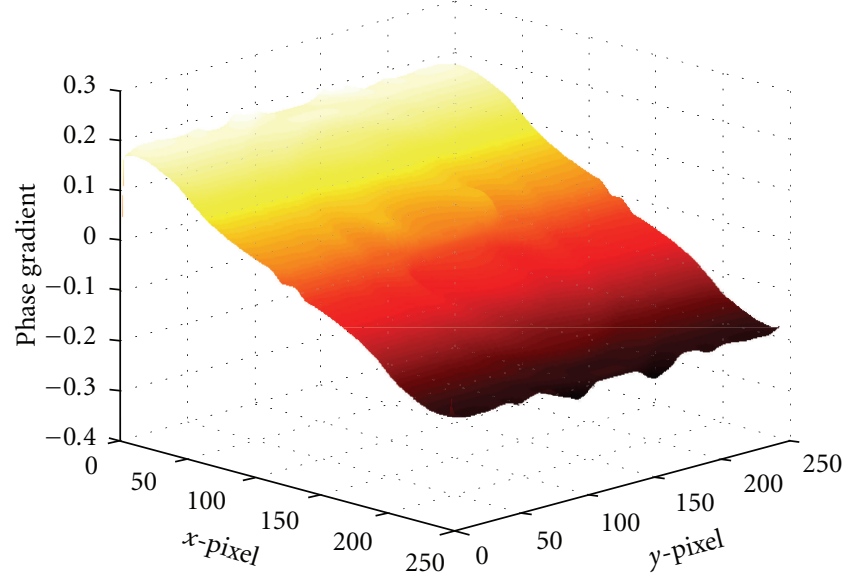

(a)

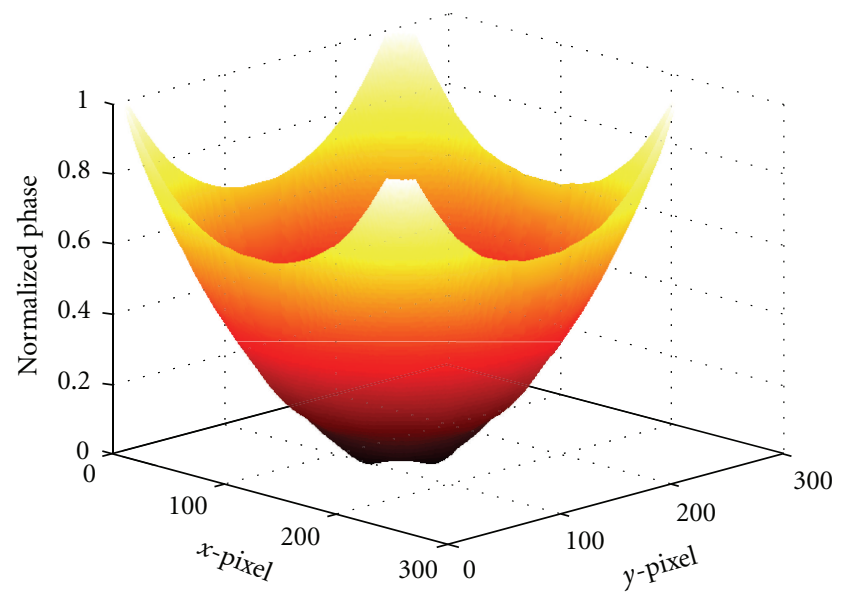

(b)

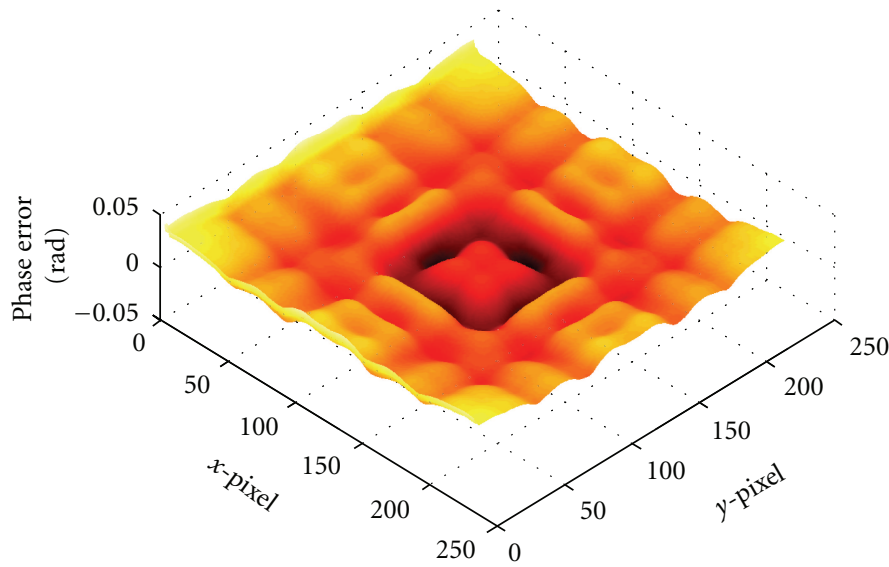

(c)

Figure 4: (a) Phase gradient in $x$ direction retrieved by (14), (b) phase distribution of (16), and (c) the difference map between the simulated and the retrieved phase.

In (9), we chose to conduct Hilbert transform on $\operatorname{IMFN}_{k}(x)$ rather than on $\operatorname{IMF}_{k}(x)$, and this to avoid the Bedrosian and Nuttall restrictions [9-12].

The expansion of the signal in terms of the functions given in (6) is

$$
\begin{gathered}
Z(x)=\sum_{k=1}^{n-1} Z_{k}(x)=\sum_{k=1}^{n-1} b_{k}(x) e^{i \phi_{k}(x)}, \\
b(x) e^{i \phi(x)}=\sum_{k=1}^{n-1} b_{k}(x) e^{i \phi_{k}(x)} .
\end{gathered}
$$

Typically, the modulation term $b(x)$ is a slowly and smoothly varying function compared to $\phi(x)$, The gradient of (12) leads to:

$$
i b(x) \frac{\partial \phi(x)}{\partial x} e^{i \phi(x)}=\sum_{k=1}^{n-1}\left[\frac{\partial b_{k}(x)}{\partial x}+i 2 \pi f_{k}(x) b_{k}(x)\right] e^{i \phi_{k}(x)}
$$

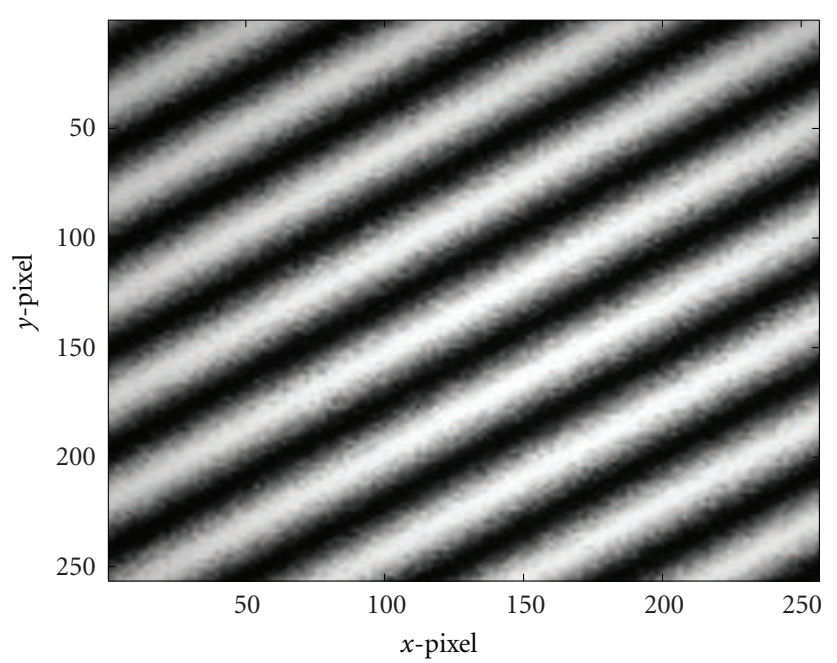

FIGURE 5: Fringe pattern for an aluminium transducer suspension assembly from a hard disk drive that was tilted between measurements. 

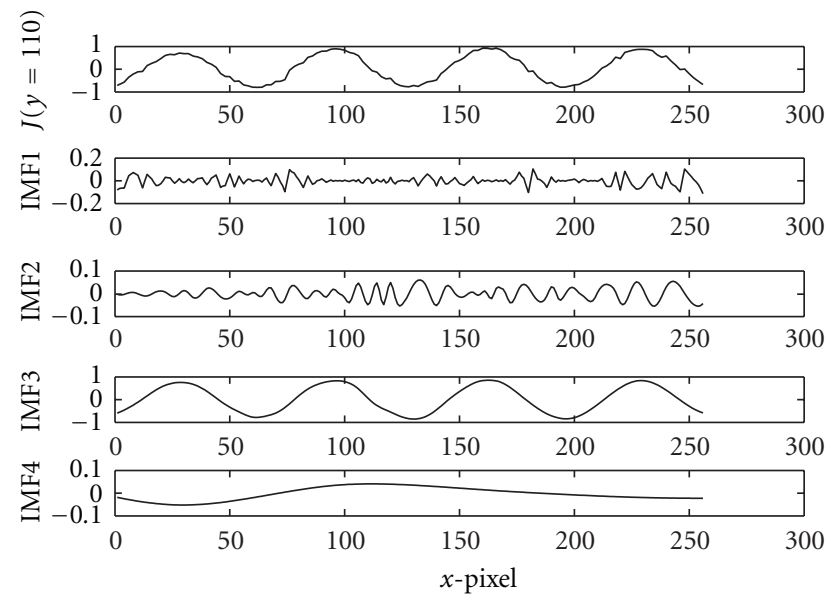

FIgURE 6: The four IMFs resulting from EMD decomposition of the data row $J(y=110)$.

Dividing (13) by (12) gives then the phase gradient by the real part $\mathfrak{R}$ of the complex expression in

$$
\frac{\partial \phi(x)}{\partial x}=\mathfrak{R}\left|\frac{\left\{\sum_{k=1}^{n-1}\left[2 \pi f_{k}(x) b_{k}(x)-i\left(\partial b_{k}(x) / \partial x\right)\right] e^{i \phi_{k}(x)}\right\}}{\left\{\sum_{k=1}^{n-1} b_{k}(x) e^{i \phi_{k}(x)}\right\}}\right|
$$

which leads to the phase by integration of the gradient.

As we can see, the $\mathrm{IMF}_{k}$ contribution to the phase gradient is

$$
\mathfrak{R}\left|\frac{\left\{\left[2 \pi f_{k}(x) b_{k}(x)-i\left(\partial b_{k}(x) / \partial x\right)\right] e^{i \phi_{k}(x)}\right\}}{\left\{\sum_{k=1}^{n-1} b_{k}(x) e^{i \phi_{k}(x)}\right\}}\right| .
$$

\section{Numerical Simulations}

The numerical simulation consists in generating digitally fringe patterns to verify the ability of the method to determine the phase distribution. The test phase function we used is

$$
\phi(x, y)=0.0009 \cdot\left[(x-128)^{2}+(y-128)^{2}\right] .
$$

The intensity distribution of fringe pattern shown in Figure 1 is

$$
I(x, y)=1+\cos (\phi(x, y)) .
$$

We illustrated in Figure 2 the IMF is extracted by EMD from a data row corresponding to $y=50$.

As we can see in Figure 3, the physical significance of each IMF is proven since we can see their individual contributions to the global phase phaset.

For determining the phase distribution of two dimensional fringe pattern, the previous procedure is generalized to all rows of the image.

The difference map between the simulated phase in Figure 4(a) and the retrieved phase in Figure 4(b) is given in Figure 4(c) where we can see that the error is small in general. Even over the large error location, the error values are smaller than $5 \%$, except for the end region, where the end effect of the Hilbert transform causes additional problems. The occasionally large error values offer an indication where the method failed. All such locations occur at the minimum amplitude. Overall, the NHHT method gives a more consistent, stable quadrature to construct the analytic signal.

\section{Experimental Results}

To illustrate the use of our method for real applications, we tested its performance on a rough ground finish, aluminium surface from a hard disk drive assembly. Changes were induced by tilting the surface and recording interferograms before and after. Figure 5 shows a fringe pattern with 9 fringes of tilt.

The 1D empirical mode decomposition method is also applied successively to reduce speckle noise in the correlation fringes produced in digital speckle pattern interferometry (DSPI) [17-19]. When decomposing a data row of the fringe pattern, it is clearly shown in Figure 6 that the first and second IMF contain only speckle noise and must be omitted to construct a smooth fringe pattern.

The results are of the same order of accuracy to DSPI measurements using the Carré algorithm [20] as it is shown in Figures 7(a) and 7(b). It is worth noting that our method gives the phase distribution with a single image and without unwrapping, while most phase evaluation methods require unwrapping phase over its definition domain, in the manner to provide a continuous phase distribution.

\section{Conclusion}

In this paper, we have presented and tested a new method of phase extraction from a single fringe pattern. This was achieved by a new exploitation of the analytic signal corresponding to each normalized intrinsic mode function. Moreover, we gave a physical sense of IMFS derived from fringe pattern decomposition by EMD. We used the NHHT since it has helped to overcome many of the difficulties of the regular 


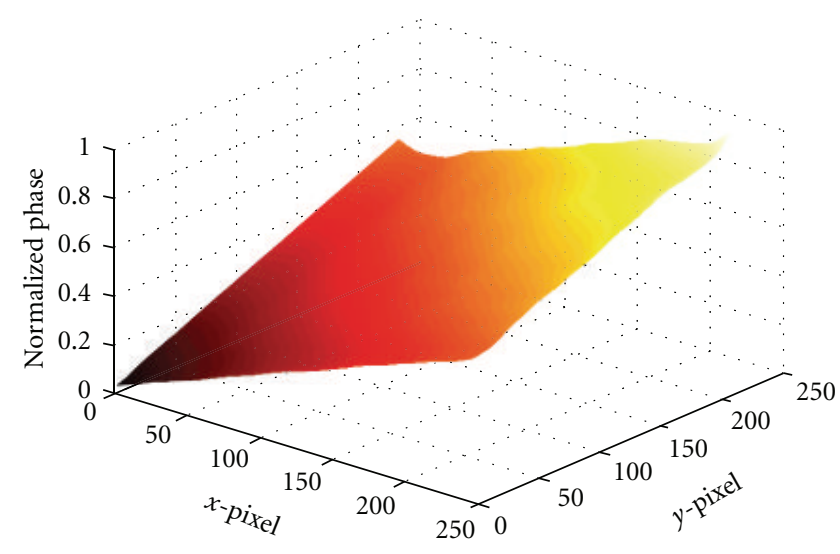

(a)

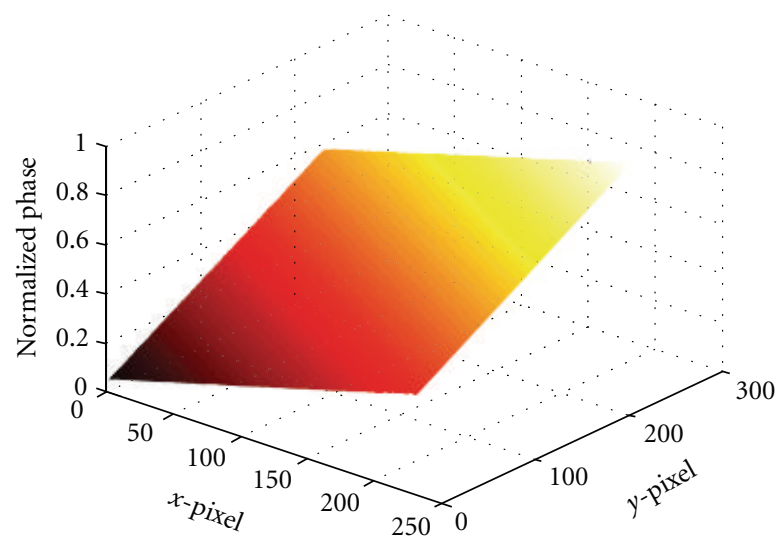

(b)

Figure 7: (a) Phase retrieved using our method, and (b) phase retrieved with Carré algorithm.

Hilbert transform, it gives consistent and stable quadrature when constructing analytic signals. The method applied to simulated and experimental fringe patterns is compared to phase-shifting methods. It shows great robustness and a very close agreement in the computation of the phase.

In summary, the advantages of this technique over other methods include the following.

(1) This method is adequate for real-time acquisition since it provides the optical phase distribution from only one single fringe pattern with a high accuracy.

(2) It is possible to perform an accurate phase demodulation on the fringe pattern even when the image is noisy.

(3) This technique requires less processing time in computation and is easy to implement.

(4) This technique leads directly to the phase distribution avoiding the complex step of phase unwrapping.

(5) The NHHT applied to fringe pattern analysis has defined the qualitative and quantitative contribution of IMFs to recover the phase. This way of fringe pattern analysis is as far as we know has not yet been reported.

\section{Acknowledgments}

The authors want to thank Dr. Joseph W. Goodman for his constructive and helpful comments. they also want to thank Dr. Neal Brock and Dr. J. C. Wyant from 4D Technology for providing them the experimental shifted fringe patterns.

\section{References}

[1] S. Sirohi and S. Chau, Optical Methods of Measurement, Marcel Dekker, New york, NY, USA, 1999.

[2] B. V. Dorrío and J. L. Fernández, "Phase-evaluation methods in whole-field optical measurement techniques," Measurement Science and Technology, vol. 10, no. 3, pp. R33-R55, 1999.
[3] D. Ghiglia and M. Pritt, Two-Dimensional Phase Unwrapping Theory, Algorithms and Applications, John Wiley \& Sons, New York, NY, USA, 1998.

[4] N. E. Huang, Z. Shen, and S. R. Long, "A new view of nonlinear water waves: the Hilbert spectrum," Annual Review of Fluid Mechanics, vol. 31, pp. 417-457, 1999.

[5] N. E. Huang, Z. Shen, S. R. Long et al., "The empirical mode decomposition and the Hubert spectrum for nonlinear and non-stationary time series analysis," Proceedings of the Royal Society, vol. 454, no. 1971, pp. 903-995, 1998.

[6] N. E. Huang and Z. Wu, "A review on Hilbert-Huang transform: method and its applications to geophysical studies," Reviews of Geophysics, vol. 46, no. 2, Article ID RG2006, 2008.

[7] N. E. Huang and S. R. Long, A generalized Zero Crossing for Local Frequency Determination, United States Patent pending, 2003.

[8] N. E. Huang, Z. Wu, S. R. Long, K. C. Arnold, X. Chen, and K. Blank, "On instantaneous frequency," Advances in Adaptive Data Analysis, vol. 1, no. 2, pp. 177-229, 2009.

[9] E. Bedrosian, "A product theorem for Hilbert transform," Proceedings of the IEEE, vol. 51, no. 2, pp. 868-869, 1972.

[10] A. H. Nuttall, "On the quadrature approximation to the Hilbert transform of modulated signals," Proceeding of IEEE, vol. 54, pp. 1458-1459, 1966.

[11] N. E. Huang and M. Bethesda, "Computing instantaneous frequency by normalizing Hilbert transform," 2005, US Patent 6901353.

[12] K. Assid, V. Dembele, F. alaoui, and A. Nassim, "Normalized Hilbert Huang transform-NHHT applied to phase extraction in wavelet domain," The Open Optics Journal, vol. 6, pp. 9-13, 2012.

[13] K. Creath, "V phase-measurement interferometry techniques," Progress in Optics, vol. 26, no. C, pp. 349-393, 1988.

[14] G. Rilling, P. Flandrin, and P. Goncalves, "On empirical mode decomposition and its algorithms," in Proceedings of IEEEEURASIP Workshop on Nonlinear Signal and Image Processing, 2003.

[15] R. C. Sharpley and V. Vatchev, "Analysis of the intrinsic mode functions," Journal of Constructive Approximation, vol. 24, no. 1, pp. 17-47, 2006.

[16] B. Boashash, "Estimating and interpreting the instantaneous frequency of a signal," Proceeding of IEEE, vol. 80, pp. 520-583, 1992. 
[17] M. B. Bernini, G. E. Galizzi, A. Federico, and G. H. Kaufmann, "Evaluation of the 1D empirical mode decomposition method to smooth digital speckle pattern interferometry fringes," Optics and Lasers in Engineering, vol. 45, no. 6, pp. 723-729, 2007.

[18] K. Assid, V. Dembele, F. alaoui, and A. Nassim, "Bidimensional empirical mode decomposition BEMD applied to speckle denoising for wavelet phase evaluation," Physical Chemical News, vol. 61, pp. 17-23, 2011.

[19] P. Flandrin, G. Rilling, and P. Gonçalvés, "Empirical mode decomposition as a filter bank," IEEE Signal Processing Letters, vol. 11, no. 2, pp. 112-114, 2004.

[20] P. Carré, "Installation et utilisation du comparateur photoélectrique et interférentiel du Bureau International des Poids et Mesures," Metrologia, vol. 2, no. 1, article 005, pp. 13-23, 1966. 

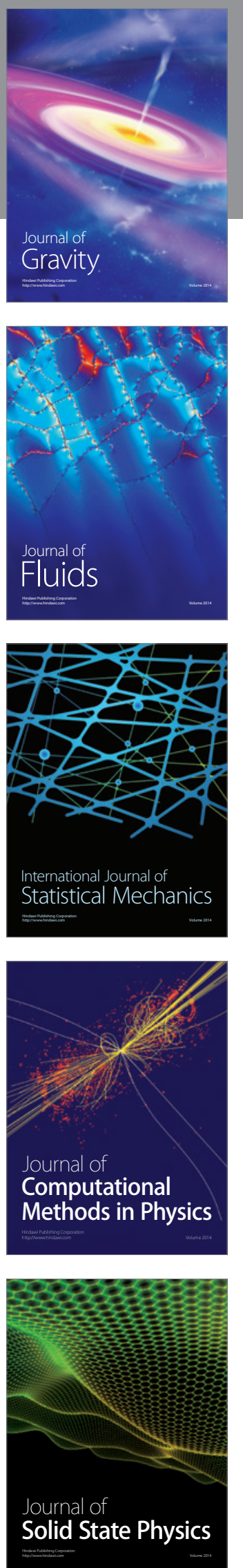
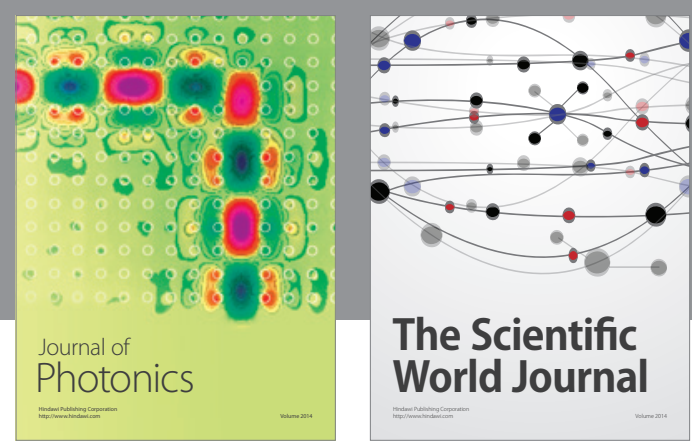

The Scientific World Journal

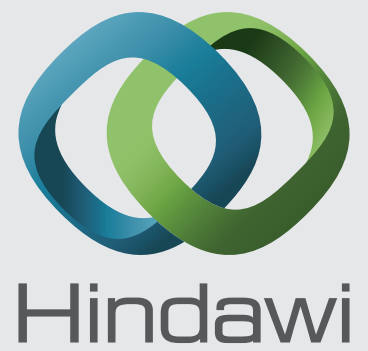

Submit your manuscripts at http://www.hindawi.com
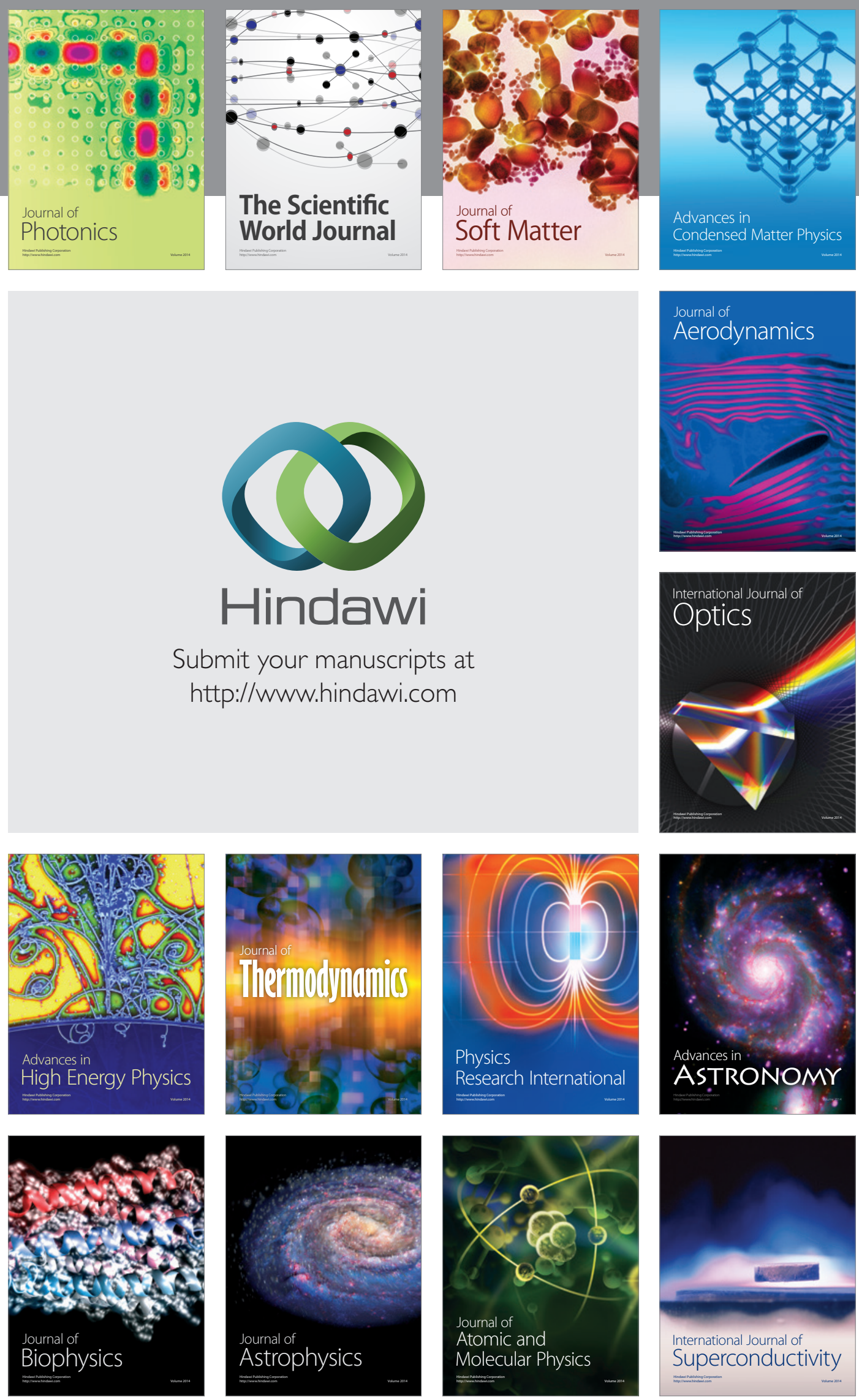
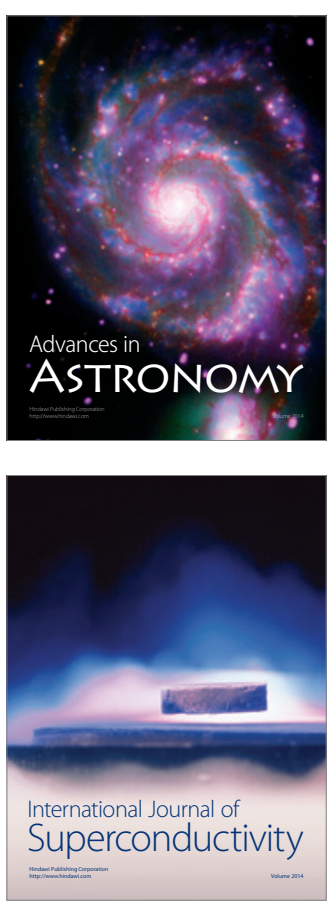